PROCEEDINGS OF THE

AMERICAN MATHEMATICAL SOCIETY

Volume 136, Number 7, July 2008, Pages 2505-2513

S 0002-9939(08)09200-9

Article electronically published on March 7, 2008

\title{
SHARP ESTIMATES \\ FOR THE IDENTITY MINUS HARDY OPERATOR ON THE CONE OF DECREASING FUNCTIONS
}

\author{
NATAN KRUGLYAK AND ERIC SETTERQVIST \\ (Communicated by N. Tomczak-Jaegermann)
}

\begin{abstract}
It is shown that if we restrict the identity minus Hardy operator on the cone of nonnegative decreasing functions $f$ in $L^{p}$, then we have the sharp estimate

for $p=2,3,4, \ldots$. In other words,

$$
\|(I-H) f\|_{L^{p}} \leq \frac{1}{(p-1)^{\frac{1}{p}}}\|f\|_{L^{p}}
$$

$$
\left\|f^{* *}-f^{*}\right\|_{L^{p}} \leq \frac{1}{(p-1)^{\frac{1}{p}}}\|f\|_{L^{p}}
$$

for each $f \in L^{p}$ and each integer $p \geq 2$.

It is also shown, via a connection between the operator $I-H$ and Laguerre functions, that

for all $\alpha \in[0,1]$.

$$
\|(1-\alpha) I+\alpha(I-H)\|_{L^{2} \rightarrow L^{2}}=\|I-\alpha H\|_{L^{2} \rightarrow L^{2}}=1
$$
\end{abstract}

\section{INTRODUCTION}

The importance of the Hardy operator

$$
(H f)(x)=\frac{1}{x} \int_{0}^{x} f(t) d t
$$

in analysis and its applications is well known. In this paper we consider the related operator

$$
(I-H) f(x)=f(x)-\frac{1}{x} \int_{0}^{x} f(t) d t,
$$

which also seems to be quite interesting. For example, in [1 it was shown that this operator is bounded and has a bounded inverse in $L^{p}\left(t^{\alpha}, \frac{d t}{t}\right)$ for $p \geq 1$ and $\alpha \in(-1, \infty) \backslash 0$. Moreover, in [2] (see also [3] for the weighted case) it was shown that if we take $e_{0}=\chi_{(0,1)}$, the characteristic function of the unit interval, then the sequence

$$
e_{n}=(I-H)^{n} e_{0}, \quad n \in \mathbb{Z}
$$

Received by the editors February 9, 2006, and, in revised form, January 26, 2007, and March 30, 2007.

2000 Mathematics Subject Classification. Primary 26D10, 46E30.

Key words and phrases. The Hardy operator, cone of decreasing functions, sharp estimates.

(C)2008 American Mathematical Society 
forms an orthonormal basis in $L^{2}=L^{2}(0, \infty)$. Therefore

$$
(I-H) e_{n}=e_{n+1}
$$

and we see that $I-H$ and its inverse $(I-H)^{-1}$ are shift isometries in $L^{2}$. In particular, we have

$$
\|(I-H) f\|_{L^{2}}=\|f\|_{L^{2}}
$$

for all $f \in L^{2}(0, \infty)$. With this interesting property in mind it is natural to consider estimates for the operator $I-H$ for the case $p \neq 2$.

This paper consists of two parts. In the first part we will show that if we consider the bijective isometry $(U f)(x)=e^{\frac{x}{2}} f\left(e^{x}\right)$ between the spaces $L^{2}(0, \infty)$ and $L^{2}(\mathbb{R})$, then the operator $U(I-H) U^{-1}$ coincides with the shift operator in the orthonormal basis in $L^{2}(\mathbb{R})$ which consists of the Laguerre functions $l_{n}(x)$ and the functions $u_{-n}(x)=l_{n}(-x)$. This gives a new simple proof of (1.1) and also shows that

$$
\|\alpha I+(1-\alpha)(H-I)\|_{L^{2} \rightarrow L^{2}}=1, \quad 0 \leq \alpha \leq 1 ;
$$

i.e., the unit sphere of the space of all bounded linear operators on $L^{2}$ contains an interval with the endpoints $I, H-I$ and the midpoint $\frac{1}{2} H$.

The second part of the paper is devoted to the study of estimates for $H-I$ and its inverse $(H-I)^{-1}$ on certain cones of functions in $L^{p}=L^{p}(0, \infty)$. To formulate the result let us consider the cone $C^{p}$ of decreasing nonnegative functions from $L^{p}$ and introduce the notation

$$
\|H-I\|_{C^{p}}=\sup _{f \in C^{p},\|f\|_{L^{p}} \leq 1}\|(H-I) f\|_{L^{p}} .
$$

We prove that

$$
\|H-I\|_{C^{p}}=\frac{1}{(p-1)^{1 / p}}, p \in\{2,3,4, \ldots\} .
$$

We give an example which shows that this estimate is not true if instead of $C^{p}$ we consider the whole space $L^{p}$. Our proof does not work for other values of $p$ and we do not know if (1.3) is true for all $p \geq 2$.

For each $f$ in the cone of decreasing functions we have

$$
(H-I) f(x)=f^{* *}(x)-f^{*}(x)
$$

for almost every $x$. So we see that (1.3) gives a sharp estimate in the following well-known equivalence in Lorentz $L^{p, q}$-spaces (see [4]):

$$
\|f\|_{L_{p, q}}:=\left(\int_{0}^{\infty}\left(x^{1 / p} f^{*}(x)\right)^{q} \frac{d x}{x}\right)^{1 / q} \approx\left(\int_{0}^{\infty}\left(x^{1 / p}\left(f^{* *}(x)-f^{*}(x)\right)\right)^{q} \frac{d x}{x}\right)^{1 / q}
$$

for the case $q=p \in\{2,3,4, \ldots\}$. In fact the functional $f^{* *}-f^{*}$ is of considerable interest in a number of contexts. One notable instance is the paper [5] (see also [4), and there are numerous more recent papers which also deal with it.

\section{The IDENTITy Minus HARDY OPERATOR AND LAGUERRE POLYNOMIALS}

It is well known that the Laguerre polynomials $L_{n}(x), n=0,1, \ldots$ (see [6]) have the following properties:

$$
L_{n}(0)=1, L_{0}(x) \equiv 1, \quad L_{n}^{\prime}(x)-L_{n}(x)=L_{n+1}^{\prime}(x) .
$$


From (2.1) it follows that

$$
\int_{0}^{x}\left(L_{n}^{\prime}(s)-L_{n}(s)\right) d s=\int_{0}^{x} L_{n+1}^{\prime}(s) d s
$$

and, therefore,

$$
L_{n}(x)-\int_{0}^{x} L_{n}(s) d s=L_{n+1}(x) .
$$

It also follows from (2.1), using integration by parts, that

$$
\int_{x}^{\infty} e^{-s} L_{m+1}(s) d s=e^{-x}\left(L_{m+1}(x)-L_{m}(x)\right) \text { for all } m \geq 0 .
$$

It is well known that the Laguerre functions

$$
l_{n}(x)=L_{n}(x) e^{-\frac{x}{2}}
$$

form an orthonormal basis in $L^{2}(0, \infty)$. Therefore the family of functions

$$
\begin{aligned}
& g_{n}(u)=l_{n-1}(u) \chi_{(0, \infty)}, n \geq 1, \\
& g_{n}(u)=-l_{-n}(-u) \chi_{(-\infty, 0)}, n \leq 0,
\end{aligned}
$$

form an orthonormal basis in $L^{2}(\mathbb{R})$. Let $\varphi(u)=e^{-u / 2} \chi_{(0, \infty)}$. Then, by applying (2.2) for $n \geq 0$ and by applying (2.3) for $n \leq-1$, we see that the operator

$$
S f=f-f * \varphi
$$

where $*$ means convolution, has the property

$$
S g_{n}=g_{n+1} \text {. }
$$

So $S$ is a shift isometry in $L^{2}(\mathbb{R})$.

Let $f \in L^{2}(0, \infty)$ and consider the operator

$$
(U f)(x)=e^{x / 2} f\left(e^{x}\right) .
$$

As

$$
\int_{0}^{\infty} f(x)^{2} d x=\int_{-\infty}^{\infty}\left(f\left(e^{x}\right) e^{\frac{x}{2}}\right)^{2} d x
$$

we obtain that $U: L^{2}(0, \infty) \rightarrow L^{2}(\mathbb{R})$ is a bijective isometry. Furthermore,

$$
\begin{aligned}
(U H f)(u)=e^{u / 2} \frac{1}{e^{u}} \int_{0}^{e^{u}} f(s) d s & =\int_{-\infty}^{u} f\left(e^{v}\right) e^{v / 2} e^{-(u-v) / 2} d v \\
& =\int_{-\infty}^{u}(U f)(v) e^{-(u-v) / 2} d v .
\end{aligned}
$$

We get $(U H f)(u)=U f * \varphi(u)$ and therefore $U(I-H) f=U f-U f * \varphi=S U f$, i.e.

or equivalently

$$
U(I-H)=S U
$$

$$
U(I-H) U^{-1}=S .
$$

As $(U f)(x)=e^{\frac{x}{2}} f\left(e^{x}\right)$ is a bijective isometry between the spaces $L^{2}(0, \infty)$ and $L^{2}(\mathbb{R})$ we immediately obtain from the last equality that the sequence

$$
e_{n}=-U^{-1} g_{n} \quad(n \in \mathbb{Z})
$$

forms an orthonormal basis in $L^{2}(0, \infty)$ and

$$
(I-H) e_{n}=e_{n+1} \quad(n \in \mathbb{Z}) .
$$


Clearly $U\left(\chi_{(0,1)}\right)=-g_{0}$ and so $e_{0}=\chi_{(0,1)}$. Thus we have obtained a new proof of the result of [2] which was mentioned in the introduction. (Cf. (1.1)).

Corollary 1. For any $0 \leq \alpha \leq 1$ we have

$$
\|\alpha I+(1-\alpha)(H-I)\|_{L^{2} \rightarrow L^{2}}=1 ;
$$

i.e. the unit sphere of the space of all bounded linear operators on $L^{2}$ contains an interval with the endpoints $I$ and $H-I$ and the midpoint $\frac{1}{2} H$.

Proof. So far $I$ has denoted the identity operator on $L^{2}(0, \infty)$. Here it will also unambiguously denote the identity operator on $\ell^{2}(\mathbb{Z})$.

Let $V$ be the shift operator on $\ell^{2}(\mathbb{Z})$. Then it is easy to check that

$$
\|\alpha I+(1-\alpha) V\|_{\ell^{2}(\mathbb{Z}) \rightarrow \ell^{2}(\mathbb{Z})}=1
$$

for each $\alpha \in[0,1]$.

Now we let $\left\{\psi_{n}\right\}_{n \in \mathbb{Z}}$ be the canonical orthonormal basis of $\ell^{2}$, i.e., $\psi_{n}=$ $\left\{\delta_{m n}\right\}_{m \in \mathbb{Z}}$ and use the fact that, obviously, $\left\{(-1)^{n} e_{n}\right\}_{n \in \mathbb{Z}}$ is an orthonormal basis for $L^{2}(0, \infty)$. We define an isometry $W$ of $\ell^{2}(\mathbb{Z})$ onto $L^{2}(0, \infty)$ by setting $W \psi_{n}=(-1)^{n} e_{n}$ for each $n \in \mathbb{Z}$. Since $(H-I)(-1)^{n} e_{n}=(-1)^{n+1} e_{n+1}$ we see that $H-I=W V W^{-1}$. Thus (2.4) follows immediately from (2.5).

Remark 1. Using the same method of proof as in Corollary 1, we can also show that

$$
\|(1-\alpha) I+\alpha(I-H)\|_{L^{2} \rightarrow L^{2}}=\|I-\alpha H\|_{L^{2} \rightarrow L^{2}}=1
$$

for all $\alpha \in[0,1]$.

\section{Operator norms of $H-I$ AND $(H-I)^{-1}$ ON CONES IN $L^{p}(0, \infty)$}

We start with the case $p=3$.

Theorem 1. $\|H-I\|_{C^{3}}=\frac{1}{\sqrt[3]{2}}$.

Proof. Let us consider the subset consisting of all simple functions in $C^{3}$. Each simple function in $C^{3}$ can be written in the form

$$
g(x)=\sum_{i=1}^{n} c_{i} \chi_{\left(0, a_{i}\right)}, c_{i} \geq 0, i=1,2, \ldots, n, 0<a_{1}<a_{2}<\ldots<a_{n},
$$

where $\chi_{(a, b)}$ denotes the characteristic function of $(a, b)$. This subset is dense in $C^{3}$; therefore to prove that $\|H-I\|_{C^{3}} \leq \frac{1}{\sqrt[3]{2}}$, it is enough to show that

$$
\|(H-I) g\|_{L^{3}} \leq \frac{1}{\sqrt[3]{2}}\|g\|_{L^{3}}
$$

holds for all simple functions $g \in C^{3}$. Straightforward calculations give:

$$
(H-I) g(x)= \begin{cases}0 & , 0<x<a_{1}, \\ \frac{c_{1} a_{1}}{x} & , a_{1}<x<a_{2}, \\ \frac{c_{1} a_{1}+c_{2} a_{2}}{x} & , a_{2}<x<a_{3}, \\ \vdots & \vdots \\ \frac{c_{1} a_{1}+c_{2} a_{2}+\ldots+c_{n} a_{n}}{x} & , a_{n}<x .\end{cases}
$$


Let us calculate the norm of $g$ :

$$
\begin{aligned}
\|g\|_{L^{3}}^{3}= & \int_{0}^{\infty}|g(x)|^{3} d x \\
= & \left(c_{1}+c_{2}+\ldots+c_{n}\right)^{3} a_{1}+\left(c_{2}+c_{3}+\ldots+c_{n}\right)^{3}\left(a_{2}-a_{1}\right)+\ldots \\
& +\left(c_{n-1}+c_{n}\right)^{3}\left(a_{n-1}-a_{n-2}\right)+c_{n}^{3}\left(a_{n}-a_{n-1}\right) .
\end{aligned}
$$

Similarly, for the norm of $\sqrt[3]{2}(H-I) g$ :

$$
\begin{aligned}
\|\sqrt[3]{2}(H-I) g\|_{L^{3}}^{3}= & \int_{0}^{\infty}|\sqrt[3]{2}(H-I) g(x)|^{3} d x \\
= & c_{1}^{3} a_{1}^{3}\left(\frac{1}{a_{1}^{2}}-\frac{1}{a_{2}^{2}}\right)+\left(c_{1} a_{1}+c_{2} a_{2}\right)^{3}\left(\frac{1}{a_{2}^{2}}-\frac{1}{a_{3}^{2}}\right)+\ldots \\
& +\left(c_{1} a_{1}+c_{2} a_{2}+\ldots+c_{n-1} a_{n-1}\right)^{3}\left(\frac{1}{a_{n-1}^{2}}-\frac{1}{a_{n}^{2}}\right) \\
& +\left(c_{1} a_{1}+c_{2} a_{2}+\ldots+c_{n-1} a_{n-1}+c_{n} a_{n}\right)^{3} \frac{1}{a_{n}^{2}} .
\end{aligned}
$$

The two expressions (3.2) and (3.3) are both homogeneous polynomials of order 3 in the variables $c_{1}, c_{2}, \ldots, c_{n}$. Thus, to compare the sizes of $\|g\|_{L^{3}}^{3}$ and $\|\sqrt[3]{2}(H-I) g\|_{L^{3}}^{3}$, we compare the coefficients of these two polynomials in Table 1 .

TABLE 1

\begin{tabular}{|l|l|c|}
\hline & $\|g\|_{L^{3}}^{3}$ & $\|\sqrt[3]{2}(H-I) g\|_{L^{3}}^{3}$ \\
\hline$c_{i}^{3}:$ & $a_{i}$ & $a_{i}$ \\
\hline$c_{i}^{2} c_{j}, i<j:$ & $3 a_{i}$ & $3 \frac{a_{i}^{2}}{a_{j}}$ \\
\hline$c_{i} c_{j}^{2}, i<j:$ & $3 a_{i}$ & $3 a_{i}$ \\
\hline$c_{i} c_{j} c_{k}, i<j<k:$ & $6 a_{i}$ & $6 \frac{a_{i} a_{j}}{a_{k}}$ \\
\hline
\end{tabular}

To obtain these coefficients we use the multinomial theorem and note that in the case of $\|g\|_{L^{3}}^{3}$, when calculating the coefficient of $c_{i} c_{j} c_{k}$, we only have to consider the contributions of the first $m$ terms of (3.2), where $m=\min \{i, j, k\}$.

Analogously, in the case of $\|\sqrt[3]{2}(H-I) g\|_{L^{3}}^{3}$, for the coefficient of $c_{i} c_{j} c_{k}$, we only have to consider the contribution of the $m$ th term in (3.3) and those after it, where this time $m=\max \{i, j, k\}$.

We observe that the coefficients for $c_{i}^{2} c_{j}$ and $c_{i} c_{j} c_{k}$ differ between $\|g\|_{L^{3}}^{3}$ and $\|\sqrt[3]{2}(H-I) g\|_{L^{3}}^{3}$. For $c_{i}^{2} c_{j}$ we have

$$
\frac{a_{i}^{2}}{a_{j}}<a_{i}
$$

because $0<a_{i}<a_{j}$ and for $c_{i} c_{j} c_{k}$ the inequality

$$
\frac{a_{i} a_{j}}{a_{k}}<a_{i}
$$


is true because $0<a_{j}<a_{k}$. Hence, all coefficients for $\|g\|_{L^{3}}^{3}$ are greater than or equal to those for $\|\sqrt[3]{2}(H-I) g\|_{L^{3}}^{3}$. Altogether this gives the inequality

$$
\|(H-I) g\|_{L^{3}} \leq \frac{1}{\sqrt[3]{2}}\|g\|_{L^{3}} .
$$

To finish the proof of the theorem we only need to note that we obtain equality in (3.4) when $g(x)=\chi_{(0, a)}$.

For a general integer $p \geq 2$ the calculations become more involved, but in principle the same method as in Theorem 1 works.

Theorem 2. $\|H-I\|_{C^{p}}=\frac{1}{(p-1)^{1 / p}}, p \in\{2,3,4, \ldots\}$.

Proof. As in Theorem 1 it suffices to consider simple functions in $C^{p}$, which can be written in the form

$$
g(x)=\sum_{i=1}^{n} c_{i} \chi_{\left(0, a_{i}\right)}, c_{i} \geq 0, i=1,2, \ldots, n, 0<a_{1}<a_{2}<\ldots<a_{n} .
$$

Calculations using the multinomial theorem show that we have the formulas shown in Table 2 for the coefficient for a general term $c_{i_{1}}^{j_{1}} c_{i_{2}}^{j_{2}} \ldots c_{i_{m}}^{j_{m}}$ in the polynomials $\|g\|_{L^{p}}^{p}$ and $\left\|(p-1)^{1 / p}(H-I) g\right\|_{L^{p}}^{p}$,

TABLE 2

\begin{tabular}{|l|l|l|}
\hline & $\|g\|_{L^{p}}^{p}$ & $\left\|(p-1)^{1 / p}(H-I) g\right\|_{L^{p}}^{p}$ \\
\hline$c_{i_{1}}^{j_{1}} c_{i_{2}}^{j_{2}} \ldots c_{i_{m}}^{j_{m}}:$ & $\frac{p !}{j_{1} ! j_{2} ! \ldots j_{m} !} a_{i_{1}}$ & $\frac{p !}{j_{1} ! j_{2} ! \ldots j_{m} !} \frac{a_{i_{1}}^{j_{1}} a_{i_{2} \ldots a_{2}}^{j_{2}} a_{i_{m}}^{j_{m}}}{a_{i_{m}}^{p-1}}$ \\
\hline
\end{tabular}

where $m$ is an integer with $1 \leq m \leq n$ and $1 \leq i_{1}<i_{2}<\ldots<i_{m} \leq n, j_{1}+j_{2}+$ $\ldots+j_{m}=p, j_{1}>0$ and $j_{2}, j_{3}, \ldots, j_{m} \geq 0$.

The fact that we have chosen $m$ arbitrarily in the above calculations of the coefficients of the multiple powers $c_{i_{1}}^{j_{1}} c_{i_{2}}^{j_{2}} \ldots c_{i_{m}}^{j_{m}}$ means that we have accounted for all possible terms in the polynomials $\|g\|_{L^{p}}^{p}$ and $\left\|(p-1)^{1 / p}(H-I) g\right\|_{L^{p}}^{p}$.

Since $j_{1}>0$, we see that

$$
\frac{a_{i_{1}}^{j_{1}} a_{i_{2}}^{j_{2}} \ldots a_{i_{m}}^{j_{m}}}{a_{i_{m}}^{p-1}} \leq a_{i_{1}}
$$

for all possible choices of $i_{1}, i_{2}, \ldots, i_{m}, j_{1}, j_{2}, \ldots, j_{m}$ and $m$. This gives the inequality

$$
\|(H-I) g\|_{L^{p}} \leq \frac{1}{(p-1)^{1 / p}}\|g\|_{L^{p}} .
$$

The case of equality is obtained when $g(x)=\chi_{(0, a)}$.

The restriction to the functions in $C^{p} \subset L^{p}(0, \infty)$ is essential for the estimate

$$
\|(H-I) f\|_{L^{p}} \leq \frac{1}{(p-1)^{1 / p}}\|f\|_{L^{p}}
$$

to be true. We illustrate this with the following example:

Example 1. Let $f(x)=\chi_{(1,3 / 2)}$. Straightforward calculations give: $\frac{1}{\sqrt[3]{2}}\|f\|_{L^{3}}=$ $\frac{1}{\sqrt[3]{4}}$ and $\|(H-I) f\|_{L^{3}}=\sqrt[3]{\frac{11}{36}}$. Hence, $\|(H-I) f\|_{L^{3}}>\frac{1}{\sqrt[3]{2}}\|f\|_{L^{3}}$. 
We now turn to an estimate for the inverse operator $(H-I)^{-1}$. As shown in [1], this operator is given by

$$
(H-I)^{-1} f(x)=\int_{x}^{\infty} \frac{f(s)}{s} d s-f(x) .
$$

Let us introduce the cone $\widetilde{C}^{p}$ defined by

Definition 1. $\widetilde{C}^{p}=\left\{f \in L^{p}(0, \infty): x f(x)\right.$ is an increasing nonnegative function $\}$.

By analogy with $C^{p}$ we define the operator norm on $\widetilde{C}^{p}$ to be:

$$
\left\|(H-I)^{-1}\right\|_{\widetilde{C}^{p}}=\sup _{f \in \widetilde{C}^{p},\|f\|_{L^{p}} \leq 1}\left\|(H-I)^{-1} f\right\|_{L^{p}} .
$$

The reason for considering the cone $\widetilde{C}^{p}$ is connected with the following fact.

Proposition 1. The operator $H-I$ maps the cone $C^{p}$ onto the cone $\widetilde{C}^{p}$ and therefore the operator $(H-I)^{-1}$ maps the cone $\widetilde{C}^{p}$ onto the cone $C^{p}$.

Proof. First of all, if $f \in C^{p}$, then

$$
(H-I) f(x)=\frac{1}{x} \int_{0}^{x} f(t) d t-f(x)=\frac{1}{x} \int_{0}^{x}(f(t)-f(x)) d t \geq 0 .
$$

Moreover, if $0<x_{1}<x_{2}$, then

$$
\begin{aligned}
x_{1}(H-I) f\left(x_{1}\right) & =x_{1}\left(\frac{1}{x_{1}} \int_{0}^{x_{1}}\left(f(t)-f\left(x_{1}\right)\right) d t\right)=x_{2}\left(\frac{1}{x_{2}} \int_{0}^{x_{1}}\left(f(t)-f\left(x_{1}\right)\right) d t\right) \\
& \leq x_{2}\left(\frac{1}{x_{2}} \int_{0}^{x_{2}}\left(f(t)-f\left(x_{2}\right)\right) d t\right)=x_{2}(H-I) f\left(x_{2}\right) .
\end{aligned}
$$

So, since the operator $H-I$ is bounded in $L^{p}$ for $p>1$, we see that it maps the cone $C^{p}$ into the cone $\widetilde{C}^{p}$. To prove that it maps onto the cone $\widetilde{C}^{p}$ it is enough to show that $(H-I)^{-1}$ maps the cone $\widetilde{C}^{p}$ into the cone $C^{p}$. Indeed, if $f \in \widetilde{C}^{p}$, then

$$
(H-I)^{-1} f(x)=\int_{x}^{\infty} f(s) \frac{d s}{s}-f(x)=\int_{x}^{\infty} \frac{s f(s)-x f(x)}{s} \frac{d s}{s} \geq 0 .
$$

Moreover, if $0<x_{1}<x_{2}$, then

$$
\begin{aligned}
(H-I)^{-1} f\left(x_{1}\right) & =\int_{x_{1}}^{\infty} \frac{s f(s)-x_{1} f\left(x_{1}\right)}{s} \frac{d s}{s} \geq \int_{x_{2}}^{\infty} \frac{s f(s)-x_{2} f\left(x_{2}\right)}{s} \frac{d s}{s} \\
& =(H-I)^{-1} f\left(x_{2}\right) .
\end{aligned}
$$

So $(H-I)^{-1} f$ is a decreasing nonnegative function. As $(H-I)^{-1}$ is bounded in $L^{p}$ for $p>1$, we obtain that $(H-I)^{-1}$ maps $\widetilde{C}^{p}$ into $C^{p}$.

Now we proceed to our main result for $\left\|(H-I)^{-1}\right\|_{\widetilde{C}^{p}}$ :

Theorem 3. $\left\|(H-I)^{-1}\right\|_{\widetilde{C}^{q}}=(q-1)^{\frac{q-1}{q}}$, where $q=p^{\prime}=\frac{p}{p-1}$ and $p \in\{2,3,4, \ldots\}$.

Proof. Let $f \in \widetilde{C}^{q}$. Then $(H-I)^{-1} f \in C^{q}$. Therefore, for the function

$$
g(x)=\lambda\left|(H-I)^{-1} f(x)\right|^{q-1} \operatorname{sgn}\left((H-I)^{-1} f(x)\right)
$$

where

$$
\lambda=\left(\int_{0}^{\infty}\left|(H-I)^{-1} f(x)\right|^{q} d x\right)^{-1 / p}
$$


we have $\|g\|_{L^{p}}=1$ and $\int_{0}^{\infty}\left((H-I)^{-1} f(x)\right) g(x) d x=\left\|(H-I)^{-1} f\right\|_{L^{q}}$.

Moreover, from (3.5) it follows that $(H-I)^{-1}=(H-I)^{*}$. Therefore, from Hölder's inequality we have

$$
\begin{gathered}
\left\|(H-I)^{-1} f\right\|_{L^{q}}=\int_{0}^{\infty}\left((H-I)^{-1} f(x)\right) g(x) d x=\int_{0}^{\infty} f(x)((H-I) g(x)) d x \\
\leq\|f\|_{L^{q}}\|(H-I) g\|_{L^{p}} \leq \frac{1}{(p-1)^{1 / p}}\|f\|_{L^{q}}\|g\|_{L^{p}}=(q-1)^{\frac{q-1}{q}}\|f\|_{L^{q}},
\end{gathered}
$$

or equivalently

$$
\left\|(H-I)^{-1}\right\|_{\widetilde{C}^{q}} \leq(q-1)^{\frac{q-1}{q}} .
$$

We only need to note that

$$
\left\|(H-I)^{-1} f\right\|_{L^{q}}=(q-1)^{\frac{q-1}{q}}\|f\|_{L^{q}}
$$

for $f(x)=\frac{1}{x} \chi_{(a, \infty)} \in \widetilde{C}^{p}, a>0$ to finish the proof.

Corollary 2. Let $f \in C^{q}$ where $q=\frac{p}{p-1}$ and $p \in\{2,3,4, \ldots\}$. Then

$$
\|f\|_{L^{q}} \leq(q-1)^{\frac{q-1}{q}}\|(H-I) f\|_{L^{q}} .
$$

Proof. From Theorem 3 above we have

$$
\left\|(H-I)^{-1} g\right\|_{L^{q}} \leq(q-1)^{\frac{q-1}{q}}\|g\|_{L^{q}}, \forall g \in \widetilde{C}^{q} .
$$

Because $H-I$ is a bijective mapping between $C^{q}$ and $\widetilde{C}^{q}$ we can write $g=(H-I) f$. We only need to substitute this expression in (3.6).

We would like to finish by stating two problems.

Problem 1. Is it true that $\|H-I\|_{C^{p}}=\frac{1}{(p-1)^{1 / p}}$ for all $p \geq 2$ ?

Problem 2. Is it true that $\|H-I\|_{L^{p} \rightarrow L^{p}}=\frac{1}{p-1}, 1<p \leq 2$ ?

Remark 2. If $\|H-I\|_{L^{p} \rightarrow L^{p}}=\frac{1}{p-1}, 1<p \leq 2$, then from the well-known result

$$
\|H\|_{L^{p} \rightarrow L^{p}}=1+\frac{1}{p-1}
$$

we will have

$$
\|H\|_{L^{p} \rightarrow L^{p}}=\|I\|_{L^{p} \rightarrow L^{p}}+\|H-I\|_{L^{p} \rightarrow L^{p}},
$$

and therefore the unit sphere of the space of all bounded linear operators on $L^{p}$ will contain an interval with the endpoints $(p-1)(H-I)$ and $I$ (compare with Corollary 1).

\section{ACKNOWLEDGEMENTS}

The authors are very thankful to the referee for valuable comments which improved the initial version of this paper. 


\section{REFERENCES}

[1] N. Kruglyak, L. Maligranda and L.-E. Persson, On an elementary approach to the fractional Hardy inequality, Proc. Amer. Math. Soc. 128 (2000), pp. 727-734. MR.1676324(2000e:26020)

[2] A. Brown, P.R. Halmos and A.L. Shields, Cesàro operators, Acta Sci. Math. (Szeged) 26 (1965), pp. 125-137. MR0187085 (32:4539)

[3] N. Kaiblinger, L. Maligranda and L.-E. Persson, Norms in weighted $L^{2}$-spaces and Hardy operators, Function Spaces (Poznan, 1998), Lecture Notes in Pure and Appl. Math., vol. 213, Marcel Dekker, New York, 2000, pp. 205-216. MR1772126 (2001g:46061)

[4] C. Bennett and R. Sharpley, Interpolation of Operators, Academic Press, Boston, MA, 1988. MR928802 (89e:46001)

[5] C. Bennett, R. DeVore and R. Sharpley, Weak- $L^{\infty}$ and BMO, Ann. of Math. (2) 113 (1981), pp. 601-611. MR621018 (82h:46047)

[6] G. Sansone, Orthogonal Functions (Revised English Edition), Dover, New York, 1991, 411 pp. MR.1118381(92e:42015)

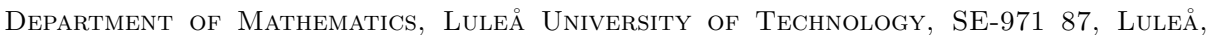
SWEDEN

E-mail address: natan@ltu.se

Global Sun Engineering AB, Aurorum Science Park 2, SE-97775 Lule̊̊, Sweden

E-mail address: eric.setterquist@globalsunengineering.com 